\title{
A Reliable Spectrum Sensing Strategy Based on Multiple-Antenna Technique
}

\author{
Shoutao $\mathrm{Lv}^{1, \mathrm{a}}$, Zeyang Dai ${ }^{1, \mathrm{~b}}$ and Jian Liu' ${ }^{2, \mathrm{c}}$ \\ ${ }^{1}$ University of Electronic Science and Technology of China (UESTC) Chengdu 611731, China \\ ${ }^{2}$ University of Science and Technology Beijing (USTB), Beijing 100083, China

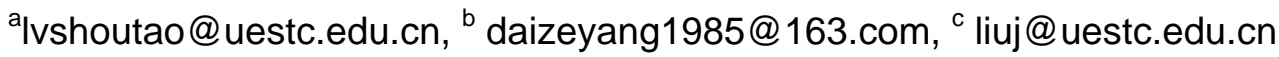

Keywords: Cognitive radio; Spectrum sensing; Multiple-antenna technique; Detection probability.

\begin{abstract}
In this paper, we propose a reliable spectrum sensing strategy based on multiple-antenna technique, called RSS-MAT, to combat the channel uncertainties. We derive the closed-form expressions of the false alarm probability and detection probability for RSS-MAT. Finally, we present simulation results to validate our performance analysis. As expected, the simulation results show that RSS-MAT outperforms the spectrum sensing strategy with single antenna.
\end{abstract}

\section{Introduction}

Spectrum sensing is a fundamental task for cognitive radio (CR). Generally, there are three sensing methods widely used in application: matched filtering detection, cyclostationary feature detection and energy detection [1, 2, 3]. However, the performances of these methods will be severely degraded by the uncertainties in wireless environments [4]. The multiple-antenna technique is an effective method to combat the channel uncertainties [5]. In [6], the authors showed the benefit of spectrum sensing using the multiple-antenna technique.

In this paper, we propose a reliable spectrum sensing strategy based on multiple-antenna technique, referred to as RSS-MAT to mitigate the effects of channel uncertainties. In RSS-MAT, the power of the signal from primary user (PU) received at each antenna is independently compared with a predefined threshold. The received signals whose powers are no less than the threshold will be amplified and added together. Finally, the secondary user (SU) uses the resultant signal to make a final decision by energy detection. We give performance analysis for RSS-MAT and derive the closed-form expressions of the false alarm and detection probabilities. Finally, simulations are presented to show that RSS-MAT achieves better performance than the single-antenna based spectrum sensing.

\section{System Model}

We consider the CR system consists of a PU $P$ and a SU $S$ equipped with $N$ antennas. The channels are modeled as independent Rayleigh fading. To improve the detection performance of $S$, RSS-MAT is proposed. Specifically, each antenna of $S$ calculates the received power from $P$ and compares it with a predefined threshold $T_{0}$. Next, $S$ amplifies the received signals whose powers are no less than $T_{0}$. Then, the amplified signals are added together at $S$. Finally, $S$ uses the resultant signal to make a final decision via energy detection.

\section{Performance Analysis}

We assume that $P$ transmits signal $x_{P}\left(E\left\{\left|x_{P}\right|^{2}\right\}=1\right)$ with power $E_{P}$. The signal to noise ratio (SNR) of $E_{P}$ is denoted as $\gamma_{P}$. We let $\theta$ denote $P$ 's state, i.e., $\theta=0$ means that $P$ is absent and $\theta=1$ means that $P$ is present. Then, the signal received at the $i$ th antenna is 


$$
y_{i}=\theta \sqrt{E_{P}} h_{i} x_{p}+n_{i}
$$

where $h_{i}$ is the channel coefficient from $P$ to the $i$ th antenna and $n_{i}$ is the additive white Gaussian noise (AWGN) with zero mean and variance $\sigma_{0}$. Clearly, $Y_{i}=\left|y_{i}\right|^{2}$ follows an exponential distribution and its expected value is given as

$$
\left\{\begin{array}{c}
\lambda_{i 0}=\sigma_{0}, H_{0} \\
\lambda_{i 1}=E_{P} \sigma_{i}+\sigma_{0}, H_{1}
\end{array}\right.
$$

where $H_{0}$ (i.e., $\theta=0$ ) and $H_{1}$ (i.e., $\theta=1$ ) are two standard test hypotheses, and $\sigma_{i}$ is the average gain of the channel $h_{i}$. According to energy detection, the false alarm and detection probabilities are

$$
\begin{aligned}
& P_{i f}=\operatorname{Pr}\left\{Y_{i} \geq T_{i} \mid H_{0}\right\}=\int_{T_{i}}^{\infty} \mathrm{e}^{-x / \lambda_{i 0}} / \lambda_{i 0} d x=\mathrm{e}^{-T_{i} / \lambda_{i 0}} \\
& P_{i d}=\operatorname{Pr}\left\{Y_{i} \geq T_{i} \mid H_{1}\right\}=\int_{T_{i}}^{\infty} \mathrm{e}^{-x / \lambda_{i 1}} / \lambda_{i 1} d x=\mathrm{e}^{-T_{i} \lambda_{i 1}}
\end{aligned}
$$

where $T_{i}$ is the power threshold. Assuming $P_{i f}=\alpha$, we have $T_{i}=-\sigma_{0} \ln (\alpha)$.

In RSS-MAT, the amplification factor is chosen as $\beta_{i}=E_{i} /\left(E_{P} \sigma_{i}+\sigma_{0}\right)$, where $E_{i}$ is set by the $i$ th antenna. The SNR of $E_{i}$ is denoted as $\gamma_{i}$. The received signal after amplified and added together is

$$
y_{S}=\sum_{i=1}^{N} \theta_{i} \sqrt{\beta_{i}} y_{i}=\theta \sum_{i=1}^{N} \theta_{i} \sqrt{\beta_{i}} \sqrt{E_{P}} h_{i} x_{p}+\sum_{i=1}^{N} \theta_{i} \sqrt{\beta_{i}} n_{i}
$$

where $\theta_{i}$ denotes the estimated value of $\theta$ made at the $i$ th antenna. In this case, the power $Y_{S}=\left|y_{S}\right|^{2}$ obeys an exponential distribution and its expected value is

$$
\left\{\begin{array}{c}
\lambda_{0}=\sum_{i=1}^{N} \theta_{i} \beta_{i} \sigma_{0}, H_{0} \\
\lambda_{1}=\sum_{i=1}^{N} \theta_{i} \beta_{i}\left(E_{P} \sigma_{i}+\sigma_{0}\right), H_{1}
\end{array}\right.
$$

Then, the false alarm and detection probabilities of RSS-MAT are respectively calculated as

$$
\begin{aligned}
& P_{f}=\operatorname{Pr}\left\{Y_{S} \geq T_{S} \mid H_{0}\right\}=\sum_{j=1}^{2^{N}-1}\left\{\left(\prod_{i \in \Phi_{j}} \alpha\right)\left(\prod_{i \in \Phi_{j}}(1-\alpha)\right)^{-T_{S} /\left(\sum_{i \in \Phi_{j}} \beta_{i} \sigma_{0}\right)}\right\} \\
& \left.P_{d}=\operatorname{Pr}\left\{Y_{S} \geq T_{S} \mid H_{1}\right\}=\sum_{j=1}^{2^{N}-1}\left\{\left(\prod_{i \in \Phi_{j}} P_{i d}\right)\left(\prod_{i \in \Phi_{j}}\left(1-P_{i d}\right)\right) \mathrm{e}^{-T_{S} / \sum_{i \in \Phi_{j}} \beta_{i}\left(E_{p} \sigma_{i}+\sigma_{0}\right)}\right]\right\}
\end{aligned}
$$

where $T_{S}$ is power threshold used by $S, \Phi_{j}$ is the $j$ th sub-collection of the set $\{1, \cdots, N\}$ and $\bar{\Phi}_{j}$ is its complementary set. Assuming $P_{f}=\alpha$, we have

$$
T_{S}=P_{f}^{-1}(\alpha)
$$


where $P_{f}^{-1}$ is the inverse function of $P_{f}$.

\section{Simulation Results}

In this section, the false alarm probability $\alpha$ is set as 0.1 . First, we consider the detection probability $P_{d}$ versus the primary transmit SNR $\gamma_{P}$ for RSS-MAT under $\sigma_{i}=0.5$ and $\sigma_{i}=1$ for $i=1,2, \cdots, N$, which are respectively illustrated in Fig. 1 and Fig. 2. Meanwhile, we choose $\gamma_{i}=1$ for $i=1,2, \cdots, N$. In Fig. 1 and Fig. 2, we also plot $P_{d}$ for the single-antenna (i.e., $N=1$ ) strategy.

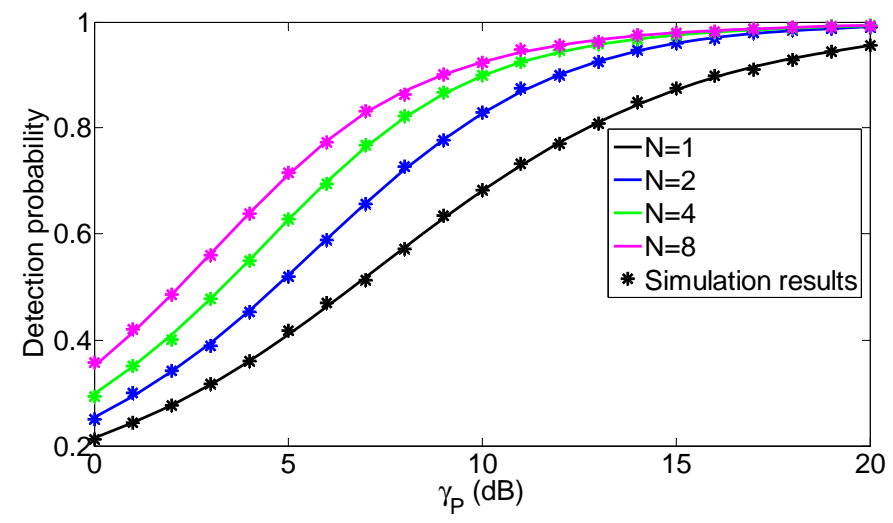

Fig. 1. $P_{d}$ versus $\gamma_{P}$ under $\sigma_{i}=0.5$.

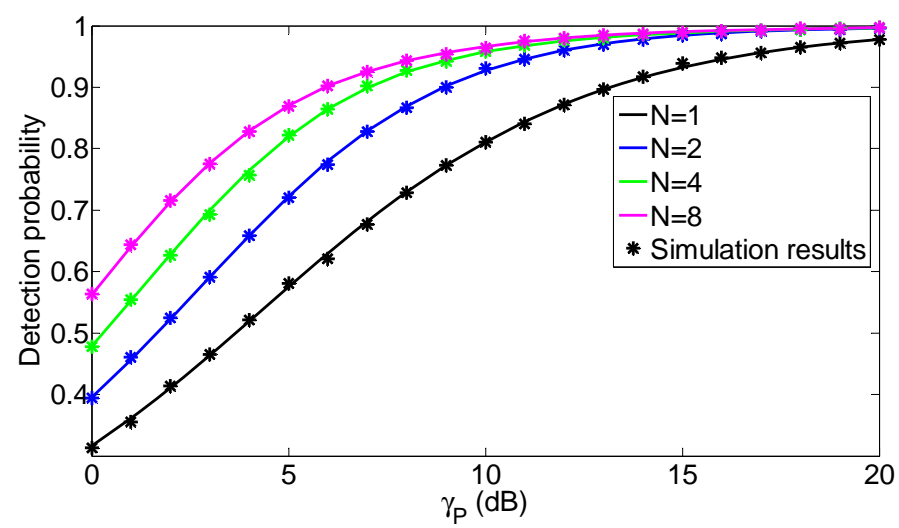

Fig. $2 P_{d}$ versus $\gamma_{P}$ under $\sigma_{i}=1$.

From Fig. 1 and Fig. 2, we can observe that RSS-MAT has higher detection probability than the single-antenna case. The detection probability of RSS-MAT increases with increasing $\gamma_{P}$. Besides, the detection performance of RSS-MAT can be improved by increasing the antenna number. Comparing Fig. 1 with Fig. 2, we also can see that better channel condition results in higher detection probability for RSS-MAT, i.e., the detection probability of RSS-MAT is higher under $\sigma_{i}=1$ than under $\sigma_{i}=0.5$.

Second, we depict $P_{d}$ versus $\gamma_{i}$ under $\sigma_{i}=0.5$ and $\sigma_{i}=1$ in Fig. 3 and Fig. 4, respectively, where we assume that $\gamma_{P}=10 \mathrm{~dB}$. The simulation results also show that RSS-MAT has better detection performance than the single-antenna case. Besides, from Fig. 3 and Fig. 4, we know that increasing $\gamma_{i}$ can not improve the detection performance of RSS-MAT significantly. 


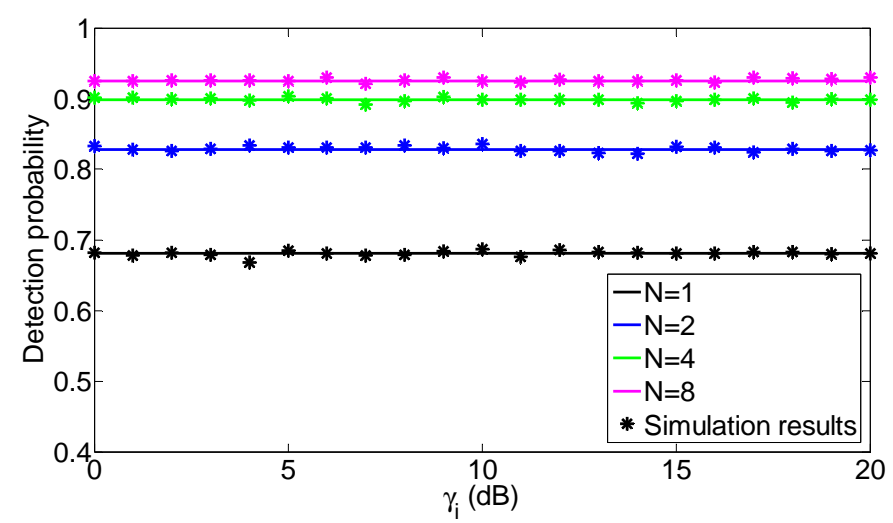

Fig. 3. $P_{d}$ versus $\gamma_{i}$ under $\sigma_{i}=0.5$.

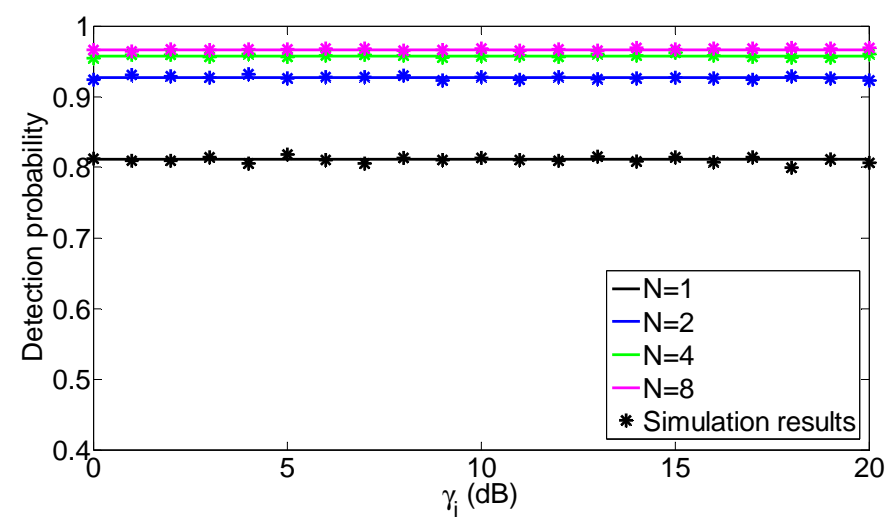

Fig. 4. $P_{d}$ versus $\gamma_{i}$ under $\sigma_{i}=1$.

\section{Summary}

In this paper, we proposed a multiple-antenna based spectrum sensing strategy, called RSS-MAT, to combat the channel uncertainties. We derive the closed-form expressions of the false alarm and detection probabilities for both the RSS-MAT and single-antenna strategies. Finally, numerical and simulation results are presented to validate the effectiveness of RSS-MAT. It is shown that RSS-MAT can achieve better detection performance than the single-antenna case. In this end, since the proposed RSS-MAT strategy has low computational complexity, it can be easily applied in practice.

\section{References}

[1] F. F. Digham, M. S. Alouini and M. K. Simon, “On the energy detection of unknown signals over fading channels,” in Proc. IEEE ICC, Anchorage, Alaska, vol. 5, pp. 3575-3579, May 2003.

[2] B. Farhang-Boroujeny, “Filter bank spectrum sensing for cognitive radios,” IEEE Trans. Signal Process., vol. 56, no. 5, pp. 1801-1811, May 2008.

[3] W. A. Gardner, "Exploitation of spectral redundancy in cyclostationary signals,” IEEE Signal Process. Mag., vol. 8, no. 2, pp. 14-36, Apr. 1991.

[4] Y. Zou, Y. Yao and B. Zheng, "A cooperative sensing based cognitive relay transmission scheme without a dedicated sensing relay channel in cognitive radio networks,” IEEE Trans. Signal Process., vol. 59, no. 2, pp. 854-858, Feb. 2011.

[5] Z. Dai, X. Wang and J. Liu, “Array calibration based on improved instrumental sensors method,” in Proc. IEEE CMC, pp. 424-428, 2011.

[6] A. Taherpour, M. Nasiri-Kenari and S. Gazor, "Multiple antenna spectrum sensing in cognitive radios,” IEEE Trans. Wireless Commun., vol. 9, no. 2, pp. 814-823, Feb. 2010. 